\title{
Cancer incidence and mortality among uranium miners in the Príbram region of the Czech Republic
}

\author{
Kaitlin Kelly-Reif ${ }^{1}{ }^{*}$, Dale Sandler ${ }^{2}$, David Shore $^{2}$, Mary Schubauer-Berigan ${ }^{3}$, Melissa \\ Troester ${ }^{1}$, Leena Nylander-French, ${ }^{1}$, and David Richardson, ${ }^{1}$ \\ ${ }^{1}$ University of North Carolina, School of Public Health, 135 Dauer Drive, NC 27599, USA \\ ${ }^{2}$ National Institute of Environmental Health Sciences, 111 TW Alexander Dr RTP, NC 27709, USA \\ ${ }^{3}$ National Institute of Occupational Safety and Health, 5555 Ridge Road Cincinnati, OH 45213, USA
}

\section{Introduction}

It is well established that underground miners exposed to radon progeny experience higher rates of lung cancer [1]. Several cohort studies of uranium miners have confirmed this, although magnitudes of associations vary somewhat between studies. Studies also suggest that radon may be associated with cancer types other than lung [1]. But, many of these findings are based on external comparison populations and may not evaluate individual radon exposure. More internal studies of dose-response relationships between radon and cancer subtypes are necessary. We investigated several subtypes of cancer incidence and mortality among underground uranium miners exposed to low and moderate levels of radon progeny.

\section{Methods}

\subsection{Cohort and case cohort studies}

A total of 16,434 male employees who worked at least 12 months underground between 1946 and 1991, and were alive and residing in Czechoslovakia on January 1, 1977 were included in the follow-up cohort [2]. Miners contributed person-time from the start of follow-up (1/1/1977) until the earliest of the date of death among deceased miners, date of migration out of the Czech Republic, or end of the study period (12/31/1992). Radon exposure in cumulative working level months (WLM) was estimated using a job exposure matrix based on company work records and area radon monitors.

A case cohort study was developed to collect additional covariate information including smoking and more precise radon doses [2]. A subcohort of 2,393 subjects was selected from the cohort by stratified random sampling on age at start of follow-up. Follow up for cancer incidence was extended through 1996.

\subsection{Statistical analysis}

* Corresponding author: kaitlink@live.unc.edu 
Standardized Mortality Ratios (SMRs), Standardized Incidence Ratios (SIRs), and associated $95 \%$ confidence intervals $(95 \% \mathrm{CI})$ were estimated by fitting a lognormal Poisson model to calculate expected cases. Expected rates were based on the national population of Czech males specific to 5-year age groups and annual calendar periods.

For internal analyses in the cohort and case cohort study, the relationship between cumulative radon exposure and cancer deaths of interest was modeled using the general model form in equation 1 .

$$
\begin{aligned}
& \text { rate }=\exp \left(a_{0}+\sum_{j=2}^{p} a_{j} x_{j}\right)\left(1+a_{1} d\right) \text { rate }=\exp \left(\mathrm{a}_{0}+\sum_{\mathrm{j}=2}^{\mathrm{p}} \mathrm{a}_{\mathrm{j}} \mathrm{x}_{\mathrm{j}}\right)\left(1+\mathrm{a}_{1} \mathrm{~d}\right) \\
& \text { rate }=\exp \left(\beta_{0}+\sum_{i=1}^{k-1} \beta_{i} d_{i}+\sum_{j=k}^{p-1} \beta_{j} x_{j}\right) \text { rate }=\exp \left(\beta_{0}+\sum_{i=1}^{k-1} \beta_{i} d_{i}+\sum_{j=k}^{p-1} \beta_{j} x_{j}\right)
\end{aligned}
$$

$\alpha_{1}$ is the excess relative rate (ERR) per unit of lagged cumulative WLM, $d . \alpha_{j}$ are parameters for effects of covariates $x_{j}$. Rate ratios by WLM category were also examined.

\section{Results}

There was a $52 \%$ increase in deaths from all malignant causes of death compared to expected rates $(\mathrm{SMR}=1.52$ 95\%CI: 1.44-1.60). Lung cancer mortality and incidence were both substantially elevated $(\mathrm{SMR}=2.12,95 \% \mathrm{CI}: 1.96-2.28$; SIR $=2.31,95 \% \mathrm{CI}: 2.15$ 2.48). Stomach cancer (SIR $=1.37,95 \% \mathrm{CI}$ : $1.11-1.63)$, rectal cancer $(\mathrm{SIR}=1.41,95 \% \mathrm{CI}$ : 1.16-1.66), liver cancer $(\mathrm{SIR}=1.70,95 \% \mathrm{CI}$ : 1.16-2.25), and extrathoracic cancer incidences ( $\mathrm{SIR}=1.41,95 \% \mathrm{CI}$ : $1.15-1.77$ ) were also in excess.

Lung cancer mortality increased with higher cumulative radon exposure $(\mathrm{ERR} / 100 \mathrm{WLM}=0.22,95 \% \mathrm{CI}: 0.10-0.37)$. Windows of exposure varied substantially, with the highest ERR in the 15-30-year window (ERR/100WLM $=0.44,95 \%$ CI: 0.21 0.67). Positive but imprecise associations between cumulative radon exposure and extrathoracic airway cancers were observed (ERR/100 WLM $=0.12,95 \% \mathrm{CI}$ : $-0.25-0.48$ ), as well as chronic lymphocytic leukemia (CLL) (ERR/100 WLM $=0.24$, 95\%CI: -0.80 1.27).

In the case cohort study, the estimated linear excess relative rate of lung cancer incidence per 100 WLM was modified by smoking status. The estimated ERR/100 WLM among non-smokers was 0.12 (95\%CI: $-0.09,0.33)$ and among smokers 1.34 (95\%CI: 0.88 , $1.80)$.

\section{Conclusions}

The Příbram miner study is a large, well enumerated cohort with available data on incidence and mortality, as well as smoking for some workers. Our results support the positive association between radon progeny and lung cancer. The results also suggest that more research is needed on prolonged exposure to low levels of radon and death from cancers other than lung, especially extrathoracic cancer and CLL.

This work was supported by the National Institute for Occupational Safety and Health, Centers for Disease Control and Prevention (T42-OH008673) and was funded in part by the Intramural Research Program of the NIH, National Institute of Environmental Health Sciences (Z01-ES049028).

\section{References}

1. National Research Council. Health Effects of Exposure to Radon. Washington, D.C.: 
National Academies Press, 1999.

2. Kulich M, Reřicha V, Reřicha $\mathrm{R}$ et al. Incidence of non-lung solid cancers in Czech uranium miners: a case-cohort study. Environ Res 2011;111:400-5. 